\title{
Exciton recombination in one-dimensional organic Mott insulators
}

\author{
Zala Lenarčič ${ }^{1,2}$ Martin Eckstein, ${ }^{3}$ and Peter Prelovšek ${ }^{2,4}$ \\ ${ }^{1}$ Institute for Theoretical Physics, University of Cologne, D-50937 Cologne, Germany \\ ${ }^{2}$ J. Stefan Institute, SI-1000 Ljubljana, Slovenia \\ ${ }^{3}$ Max Planck Research Department for Structural Dynamics, University of Hamburg-CFEL, D-22761 Hamburg, Germany \\ ${ }^{4}$ Faculty of Mathematics and Physics, University of Ljubljana, SI-1000 Ljubljana, Slovenia
}

(Received 17 August 2015; published 10 November 2015)

\begin{abstract}
We present a theory for the recombination of (charged) holons and doublons in one-dimensional organic Mott insulators, which is responsible for the decay of the photoexcited state. Due to the charge-spin separation, the dominant mechanism for recombination at low density of charges involves a multiphonon emission. We show that a reasonable coupling to phonons is sufficient to explain the fast recombination observed by pumpprobe experiments in $\mathrm{ET}_{-} \mathrm{F}_{2} \mathrm{TCNQ}$, whereby we can also account for the measured pressure dependence of the recombination rate.
\end{abstract}

DOI: 10.1103/PhysRevB.92.201104

PACS number(s): 71.27.+a, 78.47.J-, 78.55.Kz

Introduction. Femtosecond pump-probe spectroscopy is a powerful probe for the charge relaxation and thermalization phenomena in complex materials. These measurements can directly address and unveil the role of strong electron correlations, as well as the coupling to phonon degrees of freedom. Materials that behave as Mott insulators due to strong electron Coulomb repulsion contain all the latter physics, and are therefore of high theoretical and experimental interest. It has been observed that photoinduced charges decay within the picosecond range, i.e., well within the experimental resolution, but on the other hand orders of magnitude faster than in clean semiconductors with similar energy gaps.

So far two classes of Mott insulators, investigated by pumpprobe spectroscopy, revealed similar behavior. These are the layered undoped cuprates $\mathrm{La}_{2} \mathrm{CuO}_{4}$ and $\mathrm{Nd}_{2} \mathrm{CuO}_{4}$ [1-3], and the quasi-one-dimensional (1D) organic Mott insulators of the tetracyanoquinodimethane (TCNQ) family [4], in particular, ET- $F_{2}$ TCNQ [4-7], which will be the focus of our study. Both undoped cuprates and ET- $F_{2}$ TCNQ reveal ultrafast picosecond charge recombination with some similarities: (a) The charged carriers created by the pump pulse above the Mott-Hubbard (MH) gap are holons and doublons, and their recombination requires the distribution of a large energy quantum (the $\mathrm{MH}$ gap $\Delta \sim 1 \mathrm{eV}$ ) into several final excitations with smaller energy $\epsilon_{0}$. At low density of charges candidates for recipient bosons can be spin or phonon excitations. (b) The decay is exponential in time. This excludes bi- and higher-molecular processes involving inelastic collision of several "free" charge carriers, and implicitly reveals the existence of an intermediate bound state of a holon and a doublon (the MH exciton). In this respect a different observation has been obtained on $\mathrm{Ca}_{2} \mathrm{CuO}_{3}$ from the $1 \mathrm{D}$ cuprate family, which is known to have negligible excitonic effects [8] and thus shows a nonexponential decay [9].

From a theoretical viewpoint the challenge of understanding the charge recombination has analogies with the decay of the double occupancy in ultracold bosons [10] and fermions $[11,12]$ in optical lattices, where the decay rate $\Gamma$ exhibits an exponential dependence on the ratio of the Coulomb repulsion $U$ and the typical excitation's energy scale $\epsilon_{0}$. In the latter case the system can be described by a high-temperature state with a sufficient density of excited charges, so that the creation of particle-hole pairs in the compressible background [12] is the dominant decay channel, and $\epsilon_{0}$ is set by the kinetic energy of recipient excitations, as observed also within dynamical mean-field theory [13]. On the other hand, in real materials the final effective temperature is low, $T \ll U$. For the case of two-dimensional (2D) undoped cuprates, which are antiferromagnets at low $T$, a theory has been presented $[14,15]$ where the fast charge recombination is explained via emission of spin excitations with the spin exchange energy, $\epsilon_{0} \sim J$, as the relevant excitation scale. Strong correlations and large $J$ at the same time lead to a nontrivial origin of the $s$-type bound state of holon and doublon [16], i.e., the MH exciton, being the intermediate state essential for the exponential decay.

In spite of similarities with 2D Mott insulators, in quasi1D Mott insulators the scenario involving spin excitations cannot be effective either for the MH exciton formation or for the multiboson emission due to the phenomenon of charge-spin separation. In the following we will show that a multiphonon emission can be a viable recombination mechanism in 1D organic Mott insulators, somewhat specific to organic materials with energetic intramolecular vibrations and strong electron-phonon coupling $[17,18]$. The mechanism bears similarity with recently proposed multiphonon exciton decay in semiconducting carbon nanotubes [19], while in standard semiconductors such a scenario seems to be inefficient [20]. The prerequisite is again the existence of the 1D MH exciton [21], which can be stable in the case of longer range Coulomb repulsion. Since the photoexcited exciton is of the odd symmetry we will show that its decay becomes allowed only due to the electron-phonon coupling. Finally we show that our scenario can explain the pressure dependence of the recombination rate established recently for $\mathrm{ET}^{-\mathrm{F}_{2}} \mathrm{TCNQ}$ [7].

The problem is tackled as follows. Treating the recombination as a perturbation, we first neglect it and compute the exciton, i.e., the lowest bound state in the sector with one doublon and one holon. We then use Fermi's golden rule in order to compute the decay of the exciton $\left|\Psi_{1}\right\rangle$ into the manifold of states $\left|\Psi_{0}^{m}\right\rangle$ that consists of the charge ground state with additional phonon excitations, which in the 
process of recombination receive the energy of the exciton. In principle, virtual hoppings give rise to the spin exchange $J=4 t^{2} / U$; however, we shall neglect it in our calculation. Such approximation is justified by the charge-spin separation specific for $1 \mathrm{D}$, which makes the scattering of charges on spins ineffective (since the hopping of holons and doublons only shifts the spin background), and by the hierarchy of energy scales $J \ll \omega_{0}, t, U, V$ for typical organic materials, where $\omega_{0}$ is the relevant phonon frequency, $t$ hopping, and $U, V$ the dominant interaction parameters.

If the Mott gap $\Delta$ is of the order of several phonon frequencies $\omega_{0}, \Delta \approx n \omega_{0}$, the $n$-phonon contribution determines the matrix element in the Fermi's golden rule expression. The electron-phonon problem is controlled by two dimensionless parameters: the coupling strength $\xi=\lambda^{2} / \omega_{0}^{2}$ (where $\lambda$ is the typical electron-phonon coupling) and the adiabaticity $t / \omega_{0}$. In the most general case, computing the exciton in the presence of electron-phonon interaction is not possible analytically. To generate the admixture of $n \gg 1$ phonons to the exciton, the coupling strength $\xi$ must be treated to higher orders. On the other hand, at least the limit $t / \omega_{0} \ll 1$ is a valid starting point for molecular vibrations in organic crystals. In this case it is convenient to rewrite the Hamiltonian using a unitary Lang-Firsov transformation $e^{S}$, which measures the phonon coordinate with respect to the equilibrium position for a given charge configuration. If the transformed exciton state $\left|\tilde{\Psi}_{1}\right\rangle$ is expanded in phonon number states, $\left|\tilde{\Psi}_{1}\right\rangle \equiv$ $\left|\tilde{\Psi}_{1}^{(0)}\right\rangle+\left|\tilde{\Psi}_{1}^{(1)}\right\rangle+\left|\tilde{\Psi}_{1}^{(2)}\right\rangle+\cdots$, the zero-phonon state $\left|\tilde{\Psi}_{1}^{(0)}\right\rangle$ is already the leading contribution in $t / \omega_{0}$ so that additional phonon dressing can be neglected. Using this approximation we will derive a compact expression for the recombination rate $\Gamma:$

$$
\begin{aligned}
\Gamma= & 4 \tilde{t}^{2}\left(\frac{1}{2}-\frac{2 \tilde{t}^{2}}{\tilde{V}^{2}}\right) \sqrt{\frac{2 \pi}{\Delta \omega_{0}}} \\
& \times \exp \left[-\frac{\Delta}{\omega_{0}} \ln \left(\frac{\Delta}{2 e \xi \omega_{0}}\right)\right]\left[1-\left(\frac{1}{2}\right)^{\Delta / \omega_{0}}\right],
\end{aligned}
$$

which can easily be compared with the experiments, taking the hopping $\tilde{t}$ and the nearest-neighbor interaction $\tilde{V}$ from independent measurements.

The model. As a model for the charge recombination in organic Mott insulators we consider the 1D extended Hubbard model, where in addition to the local Hubbard repulsion $U$ and the nearest-neighbor electron hopping, a nearest-neighbor Coulomb repulsion $V>0$ is included. The latter is essential to stabilize the exciton state in 1D [21]. The Hamiltonian is split in the hopping $H_{t}$ of doublons and holons, the recombination term $H_{r c}$, and the interaction term $H_{U}$, which are written as

$$
\begin{gathered}
H_{t}=-t \sum_{\langle i j\rangle, s}\left(d_{i, s}^{\dagger} d_{j, s}-h_{i, s}^{\dagger} h_{j, s}+\text { H.c. }\right), \\
H_{r c}=-t \sum_{\langle i j\rangle, s}\left(h_{i, \bar{s}} d_{j, s}+h_{j, s} d_{i, s}+\text { H.c. }\right), \\
H_{U}=U \sum_{i} \frac{n_{i}^{d}+n_{i}^{h}}{2}+V \sum_{\langle i j\rangle} \bar{n}_{i} \bar{n}_{j},
\end{gathered}
$$

with holon and doublon creation operators $h_{i, s}^{\dagger}=c_{i, s}(1-$ $\left.n_{i, \bar{s}}\right), d_{i, s}^{\dagger}=c_{i, \bar{s}}^{\dagger} n_{i, s}$, holon and doublon density operators $n_{i}^{h}=$ $\frac{1}{2} \sum_{s} h_{i, s}^{\dagger} h_{i, s}, n_{i}^{d}=\frac{1}{2} \sum_{s} d_{i, s}^{\dagger} d_{i, s}$, and $\bar{n}_{i}=n_{i}^{d}-n_{i}^{h}$. Here $\langle i j\rangle$ denotes nearest-neighbor pairs and $\bar{s}$ the spin opposite to $s$. In addition, a generally nonlocal coupling between the charge density and dispersive phonons is introduced:

$$
H_{e p}=\sum_{j, q} \lambda_{q} e^{-i q j}\left(a_{q}^{\dagger}+a_{-q}\right) \bar{n}_{j}, \quad H_{p h}=\sum_{q} \omega_{q} a_{q}^{\dagger} a_{q} .
$$

We use $\hbar=a_{0}=1, a_{0}$ being the intersite distance.

Lang-Firsov transformation. The derivation of the standard Lang-Firsov transformation for the present case follows Ref. [15] and is presented in the Supplemental Material [22]. The exact transformed Hamiltonian is given by

$$
\begin{aligned}
\tilde{H}_{0}= & -\tilde{t} \sum_{\langle i j\rangle, s}\left(d_{i s}^{\dagger} d_{j s} e^{A_{j i}^{\dagger}} e^{A_{i j}}-h_{i s}^{\dagger} h_{j s} e^{A_{i j}^{\dagger}} e^{A_{j i}}+\text { H.c. }\right) \\
& +\tilde{U} \sum_{j} \frac{n_{j}^{d}+n_{j}^{h}}{2}+\tilde{V} \sum_{\langle i j\rangle} \bar{n}_{i} \bar{n}_{j}+\sum_{q} \omega_{q} a_{q}^{\dagger} a_{q}, \\
\tilde{H}_{r c}= & -\tilde{t} \sum_{\langle i j\rangle, s}\left(h_{i s} d_{j \bar{s}} e^{A_{j i}^{\dagger}} e^{A_{i j}}+h_{j s} d_{i \bar{s}} e^{A_{i j}^{\dagger}} e^{A_{j i}}+\text { H.c. }\right),
\end{aligned}
$$

where $A_{j j^{\prime}}^{\dagger}=\sum_{q}\left(\lambda_{q} / \omega_{q}\right)\left(e^{-i q j^{\prime}}-e^{-i q j}\right) a_{q}^{\dagger}$ is a phonon creation term, and $\tilde{U}, \tilde{V}$, and $\tilde{t}$ are renormalized interaction and hopping parameters. As shown in the Supplemental Material, corrections to the bare $U, V, t$ are given by $\tilde{U}=U-\tilde{\epsilon}_{0}$ and $\tilde{V}=V-\tilde{\epsilon}_{1}$ with $\tilde{\epsilon}_{i-j}=2 \sum_{q}\left(\left|\lambda_{q}\right|^{2} / \omega_{q}\right) \cos [q(i-j)]$, while longer range interaction shall be neglected. Bare hopping $t$ is rescaled as $\tilde{t}=t e^{-\xi_{1}}, \xi_{1}=\sum_{q}\left(\left|\lambda_{q}\right|^{2} / \omega_{q}^{2}\right)[1-\cos (q)]$.

Below we will express all results in terms of the renormalized parameters $\tilde{U}, \tilde{V}$, and $\tilde{t}$, which are determined experimentally by a fit to the linear absorption spectrum and for materials considered satisfy the condition $\tilde{U} \gg \tilde{t}$.

Exciton ground state. We now construct the ground state for the Hamiltonian $\tilde{H}_{0}$. To neglect additional phonon dressing, as explained above valid for $\tilde{t} / \omega_{0} \ll 1, \tilde{H}_{0}$ is projected to the phonon vacuum, $\tilde{H}_{0}^{(0)} \equiv\left|0_{p h}\right\rangle\left\langle 0_{p h}\left|\tilde{H}_{0}\right| 0_{p h}\right\rangle\left\langle 0_{p h}\right|$. We first construct a basis of all holon-doublon states with an arbitrary spin configuration of the remaining sites, analogous to the squeezed spin state [23]. For a given spin configuration $\underline{\sigma}=$ $\left\{\sigma_{1}, \ldots, \sigma_{L-2}\right\}$ (with $\sigma_{j}=\uparrow, \downarrow$ ), we define $\left|\underline{\sigma}^{m, j}\right\rangle$ as the state obtained by distributing the spins $\underline{\sigma}$ on lattice sites $\{1, \ldots, L\} \backslash$ $\{m, j\}$. For $m<j$

$$
\begin{aligned}
\left|\underline{\sigma}^{m, j}\right\rangle= & c_{1, \sigma_{1}}^{\dagger} \cdots c_{m-1, \sigma_{m-1}}^{\dagger} \times c_{m+1, \sigma_{m}}^{\dagger} \cdots c_{j-1, \sigma_{j-2}}^{\dagger} \\
& \times c_{j+1, \sigma_{j-1}}^{\dagger} \cdots c_{L, \sigma_{L-2}}^{\dagger}|0\rangle,
\end{aligned}
$$

and for $j<m$ analogous. We then define the holon-doublon state $\left|\underline{\sigma}_{j}^{m}\right\rangle$ by placing a doublon at site $j$,

$$
\left|\underline{\sigma}_{j}^{m}\right\rangle= \begin{cases}c_{j, \uparrow}^{\dagger} c_{j, \downarrow}^{\dagger}\left|\underline{\sigma}^{m, j}\right\rangle & \text { if } m \neq j, \\ 0 & \text { if } m=j .\end{cases}
$$

These are used to define the state with a holon-doublon pair $\left|\Phi_{j}^{m}\right\rangle$ and the state with two holons $\left|\Phi^{m, j}\right\rangle$ on an arbitrary spin background which is a superposition or mixture of 
configurations $\underline{\sigma}$,

$$
\left|\Phi_{j}^{m}\right\rangle \equiv \sum_{\underline{\sigma}} \Phi_{\underline{\sigma}}\left|\underline{\sigma}_{j}^{m}\right\rangle, \quad\left|\Phi^{m, j}\right\rangle \equiv \sum_{\underline{\sigma}} \Phi_{\underline{\sigma}}\left|\underline{\sigma}^{m, j}\right\rangle .
$$

One can see that the Hamiltonian $\tilde{H}_{0}^{(0)}$ does not mix different configurations $\underline{\sigma}$, because nearest-neighbor hopping of a holon or doublon implies a shift of the spin background, which is implicit in the definition (9) for $\left|\underline{\sigma}_{j}^{i}\right\rangle \rightarrow\left|\underline{\sigma}_{j}^{i \pm 1}\right\rangle$ or $\left|\underline{\sigma}_{j}^{i}\right\rangle \rightarrow$ $\left|\underline{\sigma}_{j \pm 1}^{i}\right\rangle$. The action of the Hamiltonian is thus obtained by

$$
\left(\tilde{H}_{0}^{(0)}-\tilde{U}+\delta_{|i-j|, 1} \tilde{V}\right)\left|\Phi_{j}^{i}\right\rangle=-\tilde{t} \sum_{\alpha= \pm 1}\left(\left|\Phi_{j}^{i+\alpha}\right\rangle-\left|\Phi_{j+\alpha}^{i}\right\rangle\right) .
$$

To determine the ground state we start from a partial Fourier transform with respect to the average position,

$$
\left|\psi_{q}^{l}\right\rangle=\frac{1}{\sqrt{L}} \sum_{j} e^{i q j+i q(l / 2)}\left|\Phi_{j+l}^{j}\right\rangle .
$$

With this the action of the Hamiltonian becomes

$$
\left(\tilde{H}_{0}^{(0)}-\tilde{U}+\delta_{|l|, 1} \tilde{V}\right)\left|\psi_{q}^{l}\right\rangle=-2 i \tilde{t}_{q} \sum_{\alpha= \pm 1} \alpha\left|\psi_{q}^{l+\alpha}\right\rangle,
$$

where $\tilde{t}_{q}=\tilde{t} \sin (q / 2)$. There is a continuum of states in the energy window $E \in\left[\tilde{U}-4 \tilde{t}_{q}, \tilde{U}+4 \tilde{t}_{q}\right]$. For $\tilde{H}_{0}^{(0)}$, parity-even and odd bound states are degenerate. We can restrict the analysis to the odd states, which can be created by the optical dipolar transition, and thus make the ansatz

$$
\left|\tilde{\Psi}_{1}^{(0)}\right\rangle=\sum_{l>0} \beta_{l}\left(\left|\psi_{q}^{l}\right\rangle-\left|\psi_{q}^{-l}\right\rangle\right)\left|0_{p h}\right\rangle, \quad \beta_{l}=\beta_{0} e^{-\kappa_{1} l} .
$$

The ground state is found for $q=\pi$ with $\beta_{l}=\beta_{0}(2 \tilde{t} / \tilde{V})^{l}$ and $E_{1}=\tilde{U}-\tilde{V}-4 \tilde{t}^{2} / \tilde{V}$, which lies below the continuum for $\tilde{V}>2 \tilde{t}$. Without the electron magnon coupling, the exciton is decoupled from the spin background, i.e., excitons for different spin wave functions are degenerate.

Exciton decay. Similarly to the problem of exciton decay in $2 \mathrm{D}[14,15]$, we establish the recombination rate using Fermi's golden rule

$$
\Gamma=2 \pi \sum_{m}\left|\left\langle\Psi_{0}^{m}\left|\tilde{H}_{r c}\right| \tilde{\Psi}_{1}^{(0)}\right\rangle\right|^{2} \delta\left(E_{0}^{m}-E_{1}\right),
$$

for transitions from previously determined exciton $\left|\tilde{\Psi}_{1}^{(0)}\right\rangle$, Eq. (14), into the charge ground state with additional phonon excitations $\left|\Psi_{0}^{m}\right\rangle$ via the recombination operator $\tilde{H}_{r c}$, Eq. (7). If written in an integral form [15], Eq. (15) becomes

$$
\Gamma=2 \operatorname{Re}\left\langle\tilde{\Psi}_{1}^{(0)}\left|\tilde{H}_{r c} P_{0} \int_{0}^{\infty} d \tau e^{i\left(\Delta-H_{p h}\right) \tau} P_{0} \tilde{H}_{r c}\right| \tilde{\Psi}_{1}^{(0)}\right\rangle,
$$

where $\Delta$ is the charge gap, $P_{0}$ is the projection to the zero charge sector, and $H_{p h}$ is the only part of $\tilde{H}_{0}$ which is active in the zero charge sector.

We first evaluate the application of $\tilde{H}_{r c}$ on the exciton. Starting from the expression $\left|\tilde{\Psi}_{1}^{(0)}\right\rangle$, Eq. (14), we can restrict the application of $\tilde{H}_{r c}$ to nearest-neighbor terms $l=1$,

$$
P_{0} \tilde{H}_{r c}\left|\Phi_{j+1}^{j}\right\rangle\left|0_{p h}\right\rangle=-\tilde{t} S_{j}\left|\Phi^{j, j+1}\right\rangle e^{A_{j+1, j}^{\dagger}}\left|0_{p h}\right\rangle,
$$

where $S_{j}=c_{j \uparrow}^{\dagger} c_{j+1 \downarrow}^{\dagger}-c_{j \downarrow}^{\dagger} c_{j+1 \uparrow}^{\dagger}$ creates a spin singlet on sites $j, j+1$, previously occupied by a holon-doublon pair. Similar, $P_{0} \tilde{H}_{r c}\left|\Phi_{j}^{j+1}\right\rangle\left|0_{p h}\right\rangle=-\tilde{t} S_{j}\left|\Phi^{j, j+1}\right\rangle e^{A_{j, j+1}^{\dagger}}\left|0_{p h}\right\rangle$. In summary,

$$
\begin{aligned}
P_{0} \tilde{H}_{r c}\left|\tilde{\Psi}_{1}^{(0)}\right\rangle= & -\tilde{t} \frac{\beta_{1}}{\sqrt{L}} \sum_{j} i(-1)^{j} S_{j}\left|\Phi^{j, j+1}\right\rangle \\
& \times\left(e^{A_{j+1, j}^{\dagger}}-e^{A_{j, j+1}^{\dagger}}\right)\left|0_{p h}\right\rangle .
\end{aligned}
$$

When this is inserted into Eq. (16), recombination rate is expressed as

$$
\Gamma=\tilde{t}^{2} \beta_{1}^{2} \sum_{d} g_{d} \Gamma_{d}^{p h}(\Delta),
$$

with a spin structure factor

$$
g_{d}=(-1)^{d} \frac{1}{L} \sum_{j}\left\langle\Phi^{j, j+1}\left|S_{j}^{\dagger} S_{j+d}\right| \Phi^{j+d, j+d+1}\right\rangle,
$$

and a phonon emission factor

$$
\begin{aligned}
\Gamma_{j-j^{\prime}}^{p h}(\Delta)= & 2 \operatorname{Re} \int_{0}^{\infty} d \tau e^{i \Delta \tau}\left\langle 0_{p h}\right|\left(e^{A_{j^{\prime}+1, j^{\prime}}}-e^{A_{j^{\prime}, j^{\prime}+1}}\right) \\
& \times e^{-i H_{p h} \tau}\left(e^{A_{j+1, j}^{\dagger}}-e^{A_{j, j+1}^{\dagger}}\right)\left|0_{p h}\right\rangle .
\end{aligned}
$$

Spin structure factor. From Eqs. (20) and (8) one can see that $g_{0}=2$ and $g_{1}=g_{-1}=1$ for an arbitrary spin configuration. For $d \geqslant 2$, Eq. (8) implies that for any spin configuration $\underline{\sigma},(-1)^{d}\left\langle\underline{\sigma}^{j, j+1}\left|S_{j}^{\dagger} S_{j+d}\right| \underline{\sigma}^{j+d, j+d+1}\right\rangle$ equals 1 if the spins $\left(\sigma_{j}, \ldots, \sigma_{j+d-1}\right)$ form an antiferromagnetic sequence $(\uparrow, \downarrow, \uparrow, \downarrow, \ldots)$ or $(\downarrow, \uparrow, \downarrow, \uparrow, \ldots)$, and 0 else. We thus have $g_{d \neq 0}=1$ for a perfect Néel antiferromagnet, and $g_{d}=0$ for $|d| \geqslant 2$ for a spin-polarized background. For a general finite temperature state we expect an exponential decay of the correlations with distance.

Boson emission factor. The matrix element in the boson factor (21) can be evaluated straightforwardly, which is done in the Supplemental Material [22]. We obtain

$$
\begin{aligned}
\Gamma_{d}^{p h}(\Delta)= & 8 \operatorname{Re} \int_{0}^{\infty} d \tau e^{i \Delta \tau} \\
& \times \sinh \left(2 \sum_{q} \frac{\left|\lambda_{q}\right|^{2}}{\omega_{q}^{2}} \cos (d q)(1-\cos q) e^{-i \omega_{q} \tau}\right) .
\end{aligned}
$$

The argument of the sinh may be written in a convenient way as an integral $2 \int d \omega e^{-i \omega \tau} f_{d}(\omega)$, with the boson coupling function

$$
f_{d}(\omega)=\sum_{q} \frac{\left|\lambda_{q}\right|^{2}}{\omega_{q}^{2}} \cos (d q)(1-\cos q) \delta\left(\omega-\omega_{q}\right) .
$$

The zeroth and first moment $\eta_{d}=\int d \omega f_{d}(\omega), \quad \Omega_{d} \eta_{d}=$ $\int d \omega \omega f_{d}(\omega)$ of these functions are related to the phononmediated long-range interaction parameters $\tilde{\epsilon}_{d}$ via $2 \Omega_{d} \eta_{d}=$ $\left(\tilde{\epsilon}_{d}-\frac{1}{2} \tilde{\epsilon}_{d-1}-\frac{1}{2} \tilde{\epsilon}_{d+1}\right)$. The time integration in Eq. (22) can be performed numerically for any kind of dispersions $\lambda_{q}, \omega_{q}$; however, for a fixed function $f_{d}$ and $\Delta / \omega_{q} \rightarrow \infty$, one can use an argument related to the central limit theorem to show that in the lowest order the result depends only on the zeroth 


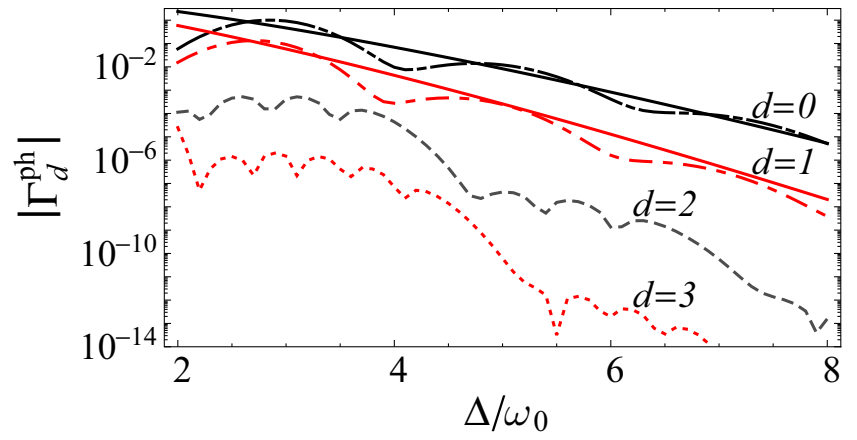

FIG. 1. (Color online) Comparison of boson emission factors $\Gamma_{d}^{p h}$ for different $d$, obtained by numerical integration of Eq. (22) for dispersions $\omega_{q}=\omega_{0}+\delta \omega \cos (q), \lambda_{q}=\lambda / \sqrt{L}$ (dashed lines) and from Eq. (24) (solid lines). Parameters $\omega_{0}=1, \delta \omega=0.1 \omega_{0}, \xi=0.3$ are used.

moment $\eta_{d}$, as presented in the Supplemental Material [22]. The integral (22) can then be approximated with

$$
\Gamma_{d}^{p h}(\Delta)=2 \frac{\left|\eta_{d}\right|}{\eta_{d}} \sqrt{\frac{2 \pi}{\Delta \omega_{0}}}\left(\frac{\Delta}{2 e\left|\eta_{d}\right| \omega_{0}}\right)^{-\Delta / \omega_{0}},
$$

where $\omega_{0}$ is the typical phonon frequency. Expression (24) is obtained also by the saddle-point approximation for a Gaussian $f_{d}(\omega)$ with the same zeroth moment $\eta_{d}$ [15].

Typically, phonons are weakly dispersive and electron phonon interactions not long ranged, so that $\left|\tilde{\epsilon}_{0}\right| \gg\left|\tilde{\epsilon}_{1}\right| \gg$ $\left|\tilde{\epsilon}_{2}\right| \ldots$. Then it suffices to take into account only the $|d| \leqslant 1$ contributions, as demonstrated in Fig. 1 for dispersions $\lambda_{q}=$ $\lambda / \sqrt{L}, \omega_{q}=\omega_{0}+\delta \omega \cos (q)$, showing results of numerical integration of Eq. (22) (dashed lines). To boost the convergence an additional smoothening $e^{-i \omega_{q} \tau} \rightarrow e^{-i \omega_{q} \tau} e^{-\eta^{2} \tau^{2} / 2}$ with $\eta=0.2 \omega_{0}$ has been used in Eq. (22), which can physically correspond to higher dimensionality of phonons or a distribution of several vibrational modes. The final expression for the recombination rate, Eq. (1), which is relevant for the comparison with experiments, is thus obtained by restricting Eq. (19) to the $|d|=0,1$ contributions with spin structure factor $g_{0}=2, g_{ \pm 1}=1$, and using approximations $\Omega_{0} \approx \Omega_{1} \approx \omega_{0}, 2 \omega_{0} \eta_{0} \approx \tilde{\epsilon}_{0}, 2 \omega_{0} \eta_{1} \approx-\tilde{\epsilon}_{0} / 2$ in Eq. (24) with $\tilde{\epsilon}_{0} \approx 2 \omega_{0} \xi$ expressed via coupling strength $\xi=\lambda^{2} / \omega_{0}^{2}$. Dependence $\Gamma_{d}^{p h}(\Delta)$, Eq. (24), with $\eta_{d}$ approximated as above is for relevant terms $d=0,1$ shown in Fig. 1 (solid lines), displaying agreement with numerical integration. Note that a nonzero $\delta \omega$ is necessary for smooth variation of $\Gamma_{d}^{p h}(\Delta)$ (otherwise recombination is possible only for discrete energies $\left.\Delta=n \omega_{0}\right)$, but after taking the asymptotic limit $\Delta / \omega_{0} \gg 1$, corrections in $\delta \omega / \omega_{0}$ to the analytical expression Eq. (24) are small at least for the dominant $|d|=0,1$, so that the latter expression is applicable for sufficient but not too large dispersion amplitudes. The prefactor $\left(1 / 2-2 \tilde{t}^{2} / \tilde{V}^{2}\right)$ in Eq. (1) comes from $\beta_{1}^{2}$.

Comparison with experiment. Finally we compare the recombination times $\tau_{r}=\Gamma^{-1}$ obtained from Eq. (1) with experimentally measured ones [7]. All quantities but the strength of charge-phonon coupling $\xi$ are set by the experimental data: $\omega_{0}=0.23 \mathrm{eV}$ [24], $\tilde{U}=0.845 \mathrm{eV}$, while $\tilde{t}, \tilde{V}$ are

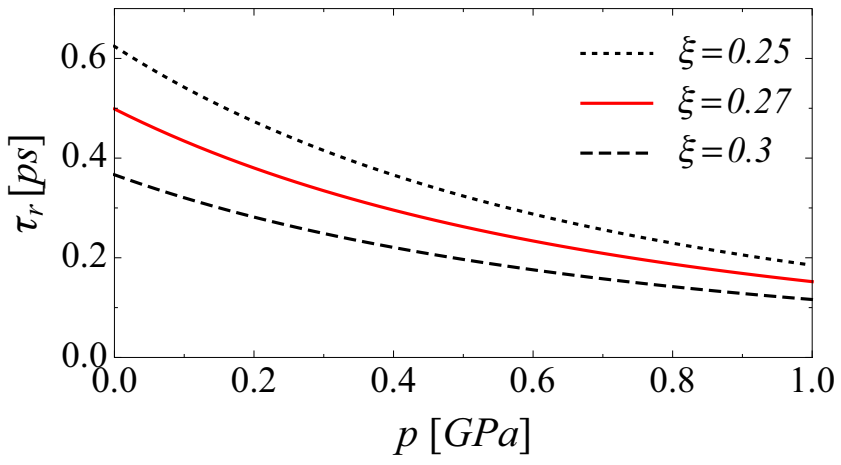

FIG. 2. (Color online) Recombination time $\tau_{r}$ as a function of pressure $p$ calculated from the Eq. (1) using the experimental parameters [7,24]: $\omega_{0}=0.23 \mathrm{eV}, U=0.845 \mathrm{eV}$, and $\tilde{t}(p) \in$ $[0,04,0.06] \mathrm{eV}, \tilde{V}(p) \in[0.12,0.16] \mathrm{eV}$ for coupling strengths $\xi=$ $0.25,0.27,0.3$.

specified functions of pressure $p, p \in[0,1] \mathrm{GPa}$, with linear dependence within intervals $\tilde{t}(p) \in[0,04,0.06] \mathrm{eV}, \tilde{V}(p) \in$ $[0.12,0.16] \mathrm{eV}[7]$, and $\Delta \approx \tilde{U}-\tilde{V}-4 \tilde{t}^{2} / \tilde{V}$.

Figure 2 displays $\tau_{r}$ as a function of pressure $p$ for three different values $\xi=0.25,0.27,0.30$, showing that $\xi \approx 0.27$ is consistent with the experimentally measured recombination times [7], yielding the electron-phonon coupling $\lambda=\omega_{0} \sqrt{\xi}=$ $0.12 \mathrm{eV}$. The latter has been measured and calculated for a similar organic material, finding $\lambda \in[0.05 \mathrm{eV}, 0.1 \mathrm{eV}]$ [17], confirming that the electron-phonon coupling needed to reproduce the experimental results is indeed realistic.

Conclusions and discussion. The central result of our study is that the fast charge recombination observed recently in quasi-1D organic Mott insulators [7] can be explained via creation of phonon excitations, which can be for the material considered (ET- $\mathrm{F}_{2} \mathrm{TCNQ}$ ) identified as molecular vibrations. Due to the charge-spin separation in 1D systems and hierarchy of energies $J<\omega_{0}$ in materials addressed, spin excitations are in contrast to 2D systems an inefficient decay channel and were neglected in our analysis by setting $J \rightarrow 0$. Motivated by the experimentally observed exponential decay of charge density we derive the recombination rate based on the assumption that a holon and a doublon initially form a bound state-exciton, which is odd under the parity transformation (therefore optically accessible). Still, the transition into the charge ground state with even parity is allowed due to the coupling to phonons. We established the charge recombination rate using Fermi's golden rule, showing approximately exponential suppression with the number of phonons emitted in the process; an observation common to several doublon decay processes with different recipients of doublon energy [11-15,25].

To understand the pressure dependence of the decay rate, not only the modulation of gap $\Delta(p)$ but also the prefactor in Eq. (1) coming from the exciton wave function should be considered. By applying the pressure the exciton is delocalized, leading to a reduction of decay rate, as recognized in Ref. [7]. While the latter reference proposed a phenomenological treatment of the decay in terms of a spin-boson model, our aim is to establish the microscopic origin of the decay mechanism, based on a more realistic 
spectral function describing coupling to vibrations that have indeed been observed in this material [24].

The experimentally established frequency of the relevant vibrations [24] is much larger than that of typical lattice phonons, making the recombination mechanism somewhat specific for organic insulators. To explain recent experiments on $1 \mathrm{D}$ cuprates $\left(\mathrm{Ca}_{2} \mathrm{CuO}_{3}\right)$ [9] with smaller typical phonon frequencies and negligible excitonic effect some modification of the mechanism might be needed and remains as a future challenge. One should note that to assist a proper dissipation of energy in the case considered the vibrations must be at least partially dispersive or coupled to other modes. Even though our derivation focuses on 1D phonons, it is straightforward to generalize it to more realistic three-dimensional electronphonon coupling. Recognizing the role of the electronphonon coupling in the recombination mechanism, we see the recombination measurements as an indirect way to establish its typically elusive strength at least for this class of materials.

Acknowledgments. The authors acknowledge helpful discussions about the experimental result with S. Kaiser, M. Mitrano, and H. Okamoto. This work has been supported by the Program P1-0044 and the project J1-4244 of the Slovenian Research Agency (ARRS). Z.L. is supported also by the L'Oréal-UNESCO national scholarship "For Women in Science."
[1] K. Matsuda, I. Hirabayashi, K. Kawamoto, T. Nabatame, T. Tokizaki, and A. Nakamura, Phys. Rev. B 50, 4097 (1994).

[2] H. Okamoto, T. Miyagoe, K. Kobayashi, H. Uemura, H. Nishioka, H. Matsuzaki, A. Sawa, and Y. Tokura, Phys. Rev. B 82, 060513 (2010).

[3] H. Okamoto, T. Miyagoe, K. Kobayashi, H. Uemura, H. Nishioka, H. Matsuzaki, A. Sawa, and Y. Tokura, Phys. Rev. B 83, 125102 (2011).

[4] H. Uemura, H. Matsuzaki, Y. Takahashi, T. Hasegawa, and H. Okamoto, J. Phys. Soc. Jpn. 77, 113714 (2008).

[5] H. Okamoto, H. Matsuzaki, T. Wakabayashi, Y. Takahashi, and T. Hasegawa, Phys. Rev. Lett. 98, 037401 (2007).

[6] S. Wall et al., Nat. Phys. 7, 114 (2011).

[7] M. Mitrano, G. Cotugno, S. R. Clark, R. Singla, S. Kaiser, J. Stähler, R. Beyer, M. Dressel, L. Baldassarre, D. Nicoletti, A. Perucchi, T. Hasegawa, H. Okamoto, D. Jaksch, and A. Cavalleri, Phys. Rev. Lett. 112, 117801 (2014).

[8] M. Ono, K. Miura, A. Maeda, H. Matsuzaki, H. Kishida, Y. Taguchi, Y. Tokura, M. Yamashita, and H. Okamoto, Phys. Rev. B 70, 085101 (2004).

[9] H. Matsuzaki, H. Nishioka, H. Uemura, A. Sawa, S. Sota, T. Tohyama, and H. Okamoto, Phys. Rev. B 91, 081114 (2015).

[10] A. L. Chudnovskiy, D. M. Gangardt, and A. Kamenev, Phys. Rev. Lett. 108, 085302 (2012).

[11] N. Strohmaier, D. Greif, R. Jördens, L. Tarruell, H. Moritz, T. Esslinger, R. Sensarma, D. Pekker, E. Altman, and E. Demler, Phys. Rev. Lett. 104, 080401 (2010).
[12] R. Sensarma, D. Pekker, E. Altman, E. Demler, N. Strohmaier, D. Greif, R. Jördens, L. Tarruell, H. Moritz, and T. Esslinger, Phys. Rev. B 82, 224302 (2010).

[13] M. Eckstein and P. Werner, Phys. Rev. B 84, 035122 (2011).

[14] Z. Lenarčič and P. Prelovšek, Phys. Rev. Lett. 111, 016401 (2013).

[15] Z. Lenarčič and P. Prelovšek, Phys. Rev. B 90, 235136 (2014).

[16] T. Tohyama, Phys. Rev. B 70, 174517 (2004).

[17] A. Girlando, J. Phys. Chem. C 115, 19371 (2011).

[18] H. Matsueda, S. Sota, T. Tohyama, and S. Maekawa, J. Phys. Soc. Jpn. 81, 013701 (2012).

[19] V. Perebeinos and P. Avouris, Phys. Rev. Lett. 101, 057401 (2008).

[20] P. Yu and M. Cardon, Fundamentals of Semiconductors: Physics and Materials Properties (Springer, Berlin, 1996).

[21] F. H. L. Essler, F. Gebhard, and E. Jeckelmann, Phys. Rev. B 64, 125119 (2001)

[22] See Supplemental Material at http://link.aps.org/supplemental/ 10.1103/PhysRevB.92.201104 for details on the derivation of the transformed Hamiltonian that explicitly show how dressing with phonon operators is obtained and simplification as well as approximate evaluation of the boson emission factor using central limit like argument and Stirling approximation.

[23] M. Ogata and H. Shiba, Phys. Rev. B 41, 2326 (1990).

[24] S. Kaiser et al., Sci. Rep. 4, 3823 (2014).

[25] R. Sensarma, D. Pekker, A. M. Rey, M. D. Lukin, and E. Demler, Phys. Rev. Lett. 107, 145303 (2011). 